\title{
RUGOSIDADE DA SUPERFÍCIE DO SOLO SOB DIFERENTES SISTEMAS DE MANEJO E INFLUENCIADA POR CHUVA $\operatorname{ARTIFICIAL}^{(1)}$
}

\author{
Elói Panachuki (2), Ildegardis Bertol ${ }^{(3)}$, Teodorico Alves Sobrinho ${ }^{(4)}$, \\ Antônio Carlos Tadeu Vitorino ${ }^{(5)}$, Cristiano Márcio Alves de Souza ${ }^{(5)} \&$ \\ Mário Artemio Urchei ${ }^{(6)}$
}

\begin{abstract}
RESUMO
A rugosidade da superfície do solo é influenciada pelo manejo, formada em especial pelo tipo de preparo e reduzida pela ação da chuva, principalmente. $O$ objetivo deste estudo foi avaliar a influência de diferentes sistemas de manejo do solo e da aplicação de chuva artificial na rugosidade da superfície do solo. Os tratamentos estudados resultaram da combinação de três sistemas de manejo do solo, semeadura direta (SD), preparo convencional (PC) e cultivo mínimo (CM), com três doses de resíduo vegetal seco de soja (Glycine max L. Merrill): 0, 2 e $4 \mathrm{Mg} \mathrm{ha}^{-1}$. Nas unidades experimentais foram aplicadas sete chuvas, com intensidade de precipitação pluvial de $60 \mathrm{~mm} \mathrm{~h}^{-1}$ e duração de $60 \mathrm{~min}$ cada, totalizando $420 \mathrm{~mm}$ de lâmina de chuva. A rugosidade foi avaliada imediatamente antes e após o preparo do solo e imediatamente após a aplicação de cada uma das sete chuvas artificiais. Obtiveram-se valores do índice de rugosidade ao acaso entre 1,88 e $5,41 \mathrm{~mm}$ na semeadura direta; entre 3,88 e $8,30 \mathrm{~mm}$ no preparo convencional; e entre 8,99 e $17,45 \mathrm{~mm}$ no cultivo mínimo. Concluiu-se que: as operações de preparo do solo aumentaram a rugosidade da sua superfície, em geral; o cultivo mínimo foi o sistema de preparo do solo que proporcionou os maiores valores de rugosidade ao acaso; e nos tratamentos semeadura direta com cobertura do solo a ação da chuva não promoveu decaimento do microrrelevo do solo.
\end{abstract}

Termos de indexação: microrrelevo superficial, manejo do solo, cobertura do solo.

\footnotetext{
(1) Parte da Tese de Doutorado do primeiro autor. Recebido para publicação em agosto de 2009 e aprovado em janeiro de 2010.

(2) Professor da Universidade Estadual de Mato Grosso do Sul - UEMS, Caixa Postal 25, CEP 79200-000 Aquidauana (MS). Email: eloip@uems.br

${ }^{(3)}$ Professor do Depto. de Solos do CAV/UDESC. CEP 88520-000 Lages (SC). Bolsista CNPq. E-mail: a2ib@cav.udesc.br

(4) Professor Associado II, Universidade Federal de Mato Grosso do Sul - CCET/UFMS. Caixa Postal 549, CEP 79070-900 Campo Grande (MS). Bolsista CNPq. E-mail: talves@ufms.br

(5) Professor da Universidade Federal da Grande Dourados - UFGD. Caixa Postal 533. E-mails: vitorino@ufgd.edu.br; csouza@ufgd.edu.br

6) Pesquisador da Embrapa Meio Ambiente, Rod. SP-340, Km 127,5, Caixa Postal 69, CEP 13820.000 Jaguariúna (SP). E-mail: urchei@cnpma.embrapa.br
} 


\title{
SUMMARY: SOIL SURFACE ROUGHNESS UNDER DIFERENT MANAGEMENT SYSTEMS AND ARTIFICIAL RAINFALL
}

\begin{abstract}
Soil roughness is influenced by soil management, particularly by soil tillage and mainly reduced by rainfall action. This study aimed to evaluate the influence of different systems of soil management and artificial rainfall application on soil surface roughness. The treatments were a result of the combination of three systems: no-tillage, conventional tillage and minimum

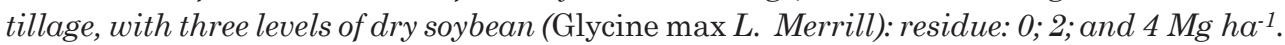
Experimental units received artificial rain (seven rains), at an intensity of $60 \mathrm{~mm} \mathrm{~h}^{-1}$ and during 60 min each, amounting to $420 \mathrm{~mm}$ rain. Roughness was evaluated immediately before and after tilling and immediately after the rainfalls. Roughness values between 1.88 and $5.41 \mathrm{~mm}$ were found under no-tillage, 3.88 and $8.30 \mathrm{~mm}$ under conventional tillage, and 3.31 and $17.45 \mathrm{~mm}$ under minimum tillage. It was concluded that soil tillage operations generally increased surface roughness. Values of random roughness were highest under minimum tillage; rain did not deteriorate the soil microrelief in the no-tillage treatments with soil cover.
\end{abstract}

Index terms: surface microrelief, soil management, soil cover.

\section{INTRODUÇÃO}

A rugosidade da superfície do solo, ou microrrelevo, refere-se às diferenças nas medidas de alturas na superfície do solo em distâncias relativamente pequenas. A rugosidade é utilizada em equações de predição de erosão hídrica e eólica e tem aplicação em hidrologia, agroclimatologia e outras áreas (Zobeck \& Popham, 1997).

Diversos fatores relacionados com as práticas de manejo do solo, com a ação do clima e com as propriedades físicas do solo influenciam a sua rugosidade superficial. Entre esses fatores, destacamse: o preparo do solo; a cobertura do solo pelos resíduos vegetais; as precipitações pluviais; o teor de água do solo antecedente ao seu preparo; a porosidade; a granulometria; e a estrutura do solo (Allmaras et al., 1967; Römkens \& Wang, 1986; Bertol et al., 2006; Taconet \& Ciarletti, 2007).

A rugosidade da superfície do solo pode, conforme Burwell et al. (1963), ser estudada considerando-se dois tipos, denominados de "rugosidade orientada" e "rugosidade ao acaso". A rugosidade orientada é caracterizada pela presença de sulcos e elevações formadas pelos implementos utilizados para preparo do solo, semeadura, aplicação de defensivos agrícolas e colheita. A rugosidade ao acaso descreve a distribuição casual dos picos e depressões da superfície do solo, não permitindo a identificação do tipo e da direção do preparo executado.

As operações de preparo do solo desempenham papel importante no potencial de erosão do solo das áreas cultivadas, alterando o microrrelevo e a cobertura por resíduos vegetais de sua camada superficial e promovendo a exposição da superfície do solo à ação da erosão hídrica, dependendo do tipo de preparo.
O microrrelevo do solo, caracterizado pela rugosidade de sua superfície, induzido pelas operações de preparo e influenciado pelos resíduos vegetais, é de fundamental importância na retenção e infiltração de água no solo, elevando a capacidade de armazenamento depressional de água na superfície e, com isso, diminuindo o escoamento superficial. A rugosidade da superfície do solo é reduzida gradativamente pela incidência das chuvas e pelo escoamento superficial em decorrência da erosão hídrica, enquanto a rugosidade criada pelos resíduos vegetais apresenta estabilidade temporal maior.

Cogo (1981) e Bertol (1995) concluíram que a presença do resíduo vegetal prolonga a permanência da rugosidade, resultando em melhor efeito na redução da erosão do solo. Isso deve-se ao fato de que a cobertura e a rugosidade superficiais são as variáveis que mais influenciam a erosão do solo, sendo responsáveis por praticamente toda a retenção e armazenagem de água e dos sedimentos da erosão na superfície do solo (Onstad, 1984; Kamphorst et al., 2000).

No Estado do Mato Grosso do Sul, as áreas de exploração agropecuária são submetidas a diferentes sistemas de manejo do solo; em razão disso, têm apresentado, nos últimos anos, uma tendência de substituição do sistema de preparo com arado ou com grade aradora combinada com grade niveladora, denominado preparo convencional, pelos sistemas conservacionistas de manejo do solo. Entretanto, ainda são raros os trabalhos de estudo da erosão do solo nessa região, especialmente quanto às alterações das propriedades da superfície do solo, como a rugosidade decorrente das operações de preparo, das quantidades e tipos de resíduos vegetais dispostos na sua superfície e da ação das chuvas. 
As considerações recém-feitas permitem avaliar a importância de se obterem mais conhecimentos sobre o tema em questão; em decorrência disso, este trabalho teve o objetivo de avaliar a influência de diferentes sistemas de manejo do solo e da aplicação de chuva artificial na rugosidade da superfície do solo.

\section{MATERIAL E MÉTODOS}

O trabalho foi realizado em área experimental da Embrapa Agropecuária Oeste, em Dourados, MS (latitude sul $22^{\circ} 14$ ', longitude $54^{\circ} 49$ 'a oeste de Greenwich e altitude média de $452 \mathrm{~m}$ ), em solo classificado como Latossolo Vermelho aluminoférrico típico, com composição granulométrica de 706 g kg-1 de argila, 94 de silte e 200 de areia. O relevo da região é plano a suavemente ondulado e a declividade média da área experimentalé de $0,03 \mathrm{~m} \mathrm{~m}^{-1}$.

Para execução do trabalho, a área experimental foi cultivada com soja (Glycine $\max \mathrm{L}$. Merrill). As avaliações de campo referentes à rugosidade da superfície do solo foram conduzidas em nove momentos diferentes, após a colheita da referida cultura, e sobre seus resíduos vegetais, permanecendo o solo, nesse período, em pousio. A primeira avaliação foi feita antes das operações de preparo do solo, e a segunda, imediatamente após essas operações. As outras sete avaliações foram efetuadas após cada chuva artificial.

As chuvas foram aplicadas com uso do simulador de chuvas desenvolvido por Alves Sobrinho et al. (2002, 2008), calibrado para aplicação de intensidade de precipitação pluvial constante e igual a $60 \mathrm{~mm} \mathrm{~h}^{-1}$, utilizando bicos Teejet 80.150 e pressão de $32 \mathrm{kPa}$. A magnitude média da energia cinética causada pela precipitação pluvial foi de $1,452 \mathrm{~kJ} \mathrm{~m}^{-2}$, estimada por meio do programa computacional Ener-Chuva, desenvolvido por Alves Sobrinho et al. (2001).

A área da parcela experimental que recebia a precipitação pluvial foi determinada por um dispositivo de formato retangular construído em chapas de aço galvanizadas $(0,7 \mathrm{~m}$ de largura, $1,0 \mathrm{~m}$ de comprimento e $0,2 \mathrm{~m}$ de altura), disposto no campo, sempre com o maior comprimento a favor do declive, até uma profundidade de $0,12 \mathrm{~m}$.

Os tratamentos estudados resultaram da combinação de três tipos de preparo do solo com três doses de resíduo vegetal seco de soja, sendo caracterizados da seguinte maneira: Trat. 1: semeadura direta sem cobertura do solo (SD-0); Trat. 2: semeadura direta com 2,0 $\mathrm{Mg} \mathrm{ha}^{-1}$ de resíduo (SD-2); Trat. 3: semeadura direta com 4,0 $\mathrm{Mg} \mathrm{ha}^{-1}$ de resíduo (SD-4); Trat. 4: preparo convencional sem cobertura do solo (PC-0); Trat. 5: preparo convencional com 2,0 $\mathrm{Mg}^{\text {ha-1 }}$ de resíduo (PC-2); Trat. 6: preparo convencional com 4,0 $\mathrm{Mg} \mathrm{ha}^{-1}$ de resíduo (PC-4); Trat. 7: cultivo mínimo sem cobertura do solo (CM-0); Trat. 8: cultivo mínimo com 2,0 Mg ha-1 de resíduo (CM-2); e Trat. 9: cultivo mínimo com 4,0 $\mathrm{Mg} \mathrm{ha}^{-1}$ de resíduo (CM-4). Os valores de resíduo vegetal seco utilizados na composição de suas doses foram obtidos com base no conceito de índice de colheita (Gomes, 2007), sendo estimada a produtividade média de $3,0 \mathrm{Mg} \mathrm{ha}^{-1}$ de grãos e utilizado o valor de 0,42 para o índice de colheita, resultando na dose de resíduo máxima de $4 \mathrm{Mg} \mathrm{ha}^{-1}$. A partir disso, foi estabelecida uma dose intermediária $\left(2,0 \mathrm{Mg} \mathrm{ha}^{-1}\right)$ e outro sem a presença de resíduo vegetal. O resíduo vegetal da área experimental foi recolhido, no momento da colheita, e conduzido até a casa de vegetação para secagem. Após uma semana, o material foi distribuído homogeneamente - de acordo com as doses propostas - em nove áreas de $25 \mathrm{~m}^{2}$ cada, onde, posteriormente, foram feitas as correspondentes operações de preparo do solo (no caso, PC e $\mathrm{CM}$ ) e instaladas duas parcelas em cada uma das áreas de distribuição dos resíduos, correspondendo a duas repetições.

$\mathrm{Na}$ área sob semeadura direta, os tratamentos foram instalados sem que houvesse revolvimento mecânico do solo. O preparo convencional consistiu de uma operação com grade aradora, de 20 discos de 0,58 m de diâmetro, seguida de duas operações com grade destorroadora niveladora, de 32 discos de $0,43 \mathrm{~m}$ de diâmetro. A profundidade efetiva de ação dos discos foi, para a grade aradora, em média, de 0,15 m e, para a grade niveladora, de $0,05 \mathrm{~m}$. O cultivo mínimo consistiu de uma operação com escarificador mecânico, seguida de uma operação com grade destorroadora niveladora. O escarificador utilizado no preparo do solo possuía sete hastes distanciadas de 0,25 m umas das outras. A profundidade média de ação das hastes foi de $0,20 \mathrm{~m}$.

O solo da área experimental foi amostrado para sua caracterização física, avaliando-se a densidade do solo e a macroporosidade (Embrapa, 1997) e o diâmetro médio geométrico de agregados (Kemper \& Rosenau, 1986) (Quadro 1).

No momento anterior ao do início dos testes foram retiradas amostras de solo, ao lado das parcelas, para verificação do teor de água no solo (Embrapa, 1997). Em cada parcela experimental, foi estimada, ainda, a percentagem de cobertura do solo pelos resíduos vegetais mediante o uso do método fotográfico com projeção de grade formada por 50 interseções sobre a fotografia, fazendo-se a contagem das interseções dos pontos desta grade com a presença ou não de cobertura vegetal nesses pontos.

Em cada tratamento, após a calibração do simulador, foram aplicadas sete chuvas com intensidade de $60 \mathrm{~mm}$ e duração de 60 min cada uma, separadas por um período de tempo de aproximadamente uma semana. As unidades experimentais permaneceram cobertas no período entre as chuvas simuladas, para evitar o efeito de chuvas naturais sobre a rugosidade da superfície do solo. 
Quadro 1. Valores médios de densidade do solo (Ds), macroporosidade (Ma) e diâmetro médio geométrico de agregados do solo (DMG), na profundidade de 0-0,05 m

\begin{tabular}{|c|c|c|c|c|c|c|}
\hline \multirow{2}{*}{ Tratamento } & \multicolumn{3}{|c|}{ Antes do preparo do solo } & \multicolumn{3}{|c|}{ Após a aplicação das chuvas } \\
\hline & Ds & Ma & DMG & Ds & Ma & DMG \\
\hline & $\mathrm{kg} \mathrm{dm}^{-3}$ & $\%$ & $\mathrm{~mm}$ & $\mathrm{~kg} \mathrm{dm}^{-3}$ & $\%$ & $\mathrm{~mm}$ \\
\hline SD - 0 & $1,30 \mathrm{ab}$ & $17,00 \mathrm{~b}$ & $4,18 \mathrm{a}$ & $1,32 \mathrm{a}$ & $16,65 \mathrm{c}$ & $4,24 \mathrm{a}$ \\
\hline SD - 2 & $1,31 \mathrm{a} \mathrm{b}$ & $15,83 \mathrm{~b}$ & $4,21 \mathrm{a}$ & $1,35 \mathrm{a}$ & $15,62 \mathrm{c}$ & $3,93 \mathrm{ab}$ \\
\hline SD - 4 & $1,44 \mathrm{a}$ & $10,56 \mathrm{c}$ & $4,40 \mathrm{a}$ & $1,29 \mathrm{ab}$ & $14,76 \mathrm{c}$ & $4,36 \mathrm{a}$ \\
\hline PC -0 & $1,26 \mathrm{~b}$ & $18,16 \mathrm{a} \mathrm{b}$ & $4,15 \mathrm{a}$ & $1,16 \mathrm{c}$ & 28,73 a & $3,19 \mathrm{c}$ \\
\hline $\mathrm{PC}-2$ & $1,35 \mathrm{ab}$ & $14,55 \mathrm{~b}$ & $4,11 \mathrm{a}$ & $1,06 \mathrm{~d}$ & $24,36 \mathrm{ab}$ & $3,91 \mathrm{ab}$ \\
\hline PC - 4 & $1,32 \mathrm{ab}$ & $16,80 \mathrm{~b}$ & $4,08 \mathrm{a}$ & $1,05 \mathrm{~d}$ & $29,04 \mathrm{a}$ & $3,22 \mathrm{c}$ \\
\hline CM - 0 & $1,24 \mathrm{~b}$ & $17,63 \mathrm{~b}$ & $4,48 \mathrm{a}$ & $1,16 \mathrm{c}$ & $25,93 \mathrm{ab}$ & $3,62 \mathrm{abc}$ \\
\hline CM - 2 & $1,26 \mathrm{~b}$ & $22,77 \mathrm{a}$ & $4,30 \mathrm{a}$ & $1,15 \mathrm{c}$ & $24,75 \mathrm{ab}$ & $3,46 \mathrm{bc}$ \\
\hline CM - 4 & $1,33 \mathrm{ab}$ & $17,39 \mathrm{~b}$ & $4,36 \mathrm{a}$ & $1,22 \mathrm{bc}$ & $22,61 \mathrm{~b}$ & $4,25 \mathrm{ab}$ \\
\hline
\end{tabular}

SD: semeadura direta; PC: preparo convencional; CM: cultivo mínimo; 0: sem cobertura do solo; 2 : com $2 \mathrm{Mg}^{-1}$ de resíduo de soja; 4: com $4 \mathrm{Mg} \mathrm{ha}^{-1}$ de resíduo de soja. Médias seguidas pela mesma letra minúscula, na mesma coluna, não diferem entre si pelo teste de Tukey a $5 \%$.

Os valores das alturas do microrrelevo do solo foram obtidos com o uso de um rugosímetro de varetas acoplado a uma máquina fotográfica digital, conforme descrito em Zoldan Junior et al. (2006). Esse instrumento é constituído de 20 varetas de $\mathrm{Al}$, alinhadas e distanciadas $30 \mathrm{~mm}$ umas das outras, que podem ser deslocadas no sentido do aclive, ocupando 20 posições, separadas de $30 \mathrm{~mm}$ uma das outras. Em cada posição, foi feito um registro fotográfico das 20 varetas verticais, gerando, com isso, um grid quadrado de $0,36 \mathrm{~m}^{2}$ com imagens das alturas de 400 varetas, para cada momento avaliado em cada parcela experimental.

Com o uso de programa computacional, cada fotografia foi digitalizada e procedeu-se à avaliação da quantidade de pixels de cada vareta, com sua posterior transformação em unidade de comprimento, correspondente à altura de cada vareta. Esse programa foi desenvolvido para obtenção de dados de rugosidade com a utilização de técnicas de análise de imagens digitalizadas. A seguir, estimou-se o índice de rugosidade ao acaso $(\mathrm{RR})$, para caracterização da rugosidade da superfície do solo.

O índice RR foi calculado como o desvio-padrão das 400 leituras das alturas do microrrelevo, sem transformação logarítmica e sem eliminar os valores extremos (10\% superiores e $10 \%$ inferiores), conforme método proposto por Kamphorst et al. (2000).

$\mathrm{Na}$ avaliação da rugosidade superficial do solo, foram considerados nove tratamentos, com duas repetições, resultando, portanto, em 18 unidades experimentais.

Os tratamentos foram dispostos em esquema de parcelas subsubdivididas, com duas repetições, segundo o delineamento inteiramente casualizado; o momento de avaliação foi considerado como parcela, o sistema de preparo, como subparcela, e a dose de resíduo vegetal, como subsubparcela. A fim de avaliar o efeito dos tratamentos e obter uma estimativa da variância residual, foi feita análise de variância dos dados, com posterior aplicação do teste de Tukey a $5 \%$, para comparação de médias. As relações entre o índice de rugosidade ao acaso e o volume de chuva acumulado foram avaliadas pelo modelo de regressão exponencial.

\section{RESULTADOS E DISCUSSÃO}

Os valores do teor de água no solo (Quadro 2), observados no momento anterior à aplicação da primeira chuva, foram relativamente baixos em todos os tratamentos, aumentando ligeiramente após a incidência das chuvas.

Considerando o mesmo momento de avaliação, observa-se que o teor de água no solo foi semelhante em todos os tratamentos estudados. Em decorrência dessa similaridade nos valores de umidade inicial do solo, pode-se considerar que o teor de água no solo não influenciou os valores da rugosidade da superfície do solo.

Com a análise dos dados (Quadro 3), verifica-se que, em geral, para um mesmo tipo de preparo, houve tendência de acréscimo no percentual de cobertura do solo com o aumento do nível de resíduo vegetal sobre a sua superfície.

Na avaliação da cobertura do solo dos três tipos de preparo, sob a mesma dose de resíduo vegetal, observase que, nos tratamentos com a presença dos resíduos, os valores do percentual de cobertura tenderam a ser, na semeadura direta, superiores aos do cultivo mínimo. Entretanto, os valores referentes ao cultivo mínimo tenderam a ser apenas ligeiramente 
Quadro 2. Teor de água do solo no momento que antecedeu a aplicação das chuvas simuladas, na profundidade de $\mathbf{0 - 0 , 0 5 ~ m ~}$

\begin{tabular}{|c|c|c|c|c|c|c|}
\hline Tratamento & ANP & $\mathrm{APP}$ & Chuva 1 & Chuva 3 & Chuva 5 & Chuva 7 \\
\hline & \multicolumn{6}{|c|}{$\mathrm{g} \mathrm{g}^{-1}$} \\
\hline SD - 0 & $0,16 \mathrm{a}$ & $0,17 \mathrm{a}$ & $0,18 \mathrm{a}$ & $0,17 \mathrm{a}$ & $0,21 \mathrm{a}$ & $0,20 \mathrm{a}$ \\
\hline $\mathrm{SD}-2$ & $0,17 \mathrm{a}$ & $0,17 \mathrm{a}$ & $0,22 \mathrm{a}$ & $0,20 \mathrm{a}$ & $0,24 \mathrm{a}$ & $0,18 \mathrm{a}$ \\
\hline $\mathrm{SD}-4$ & $0,15 \mathrm{a}$ & $0,18 \mathrm{a}$ & $0,22 \mathrm{a}$ & $0,22 \mathrm{a}$ & $0,24 \mathrm{a}$ & $0,19 \mathrm{a}$ \\
\hline PC - 0 & $0,17 \mathrm{a}$ & $0,16 \mathrm{a}$ & $0,20 \mathrm{a}$ & $0,18 \mathrm{a}$ & $0,23 \mathrm{a}$ & $0,16 \mathrm{a}$ \\
\hline $\mathrm{PC}-2$ & $0,16 \mathrm{a}$ & $0,16 \mathrm{a}$ & $0,20 \mathrm{a}$ & $0,18 \mathrm{a}$ & $0,20 \mathrm{a}$ & $0,18 \mathrm{a}$ \\
\hline PC - 4 & $0,15 \mathrm{a}$ & $0,15 \mathrm{a}$ & $0,18 \mathrm{a}$ & $0,19 \mathrm{a}$ & $0,20 \mathrm{a}$ & $0,20 \mathrm{a}$ \\
\hline CM - 0 & $0,17 \mathrm{a}$ & $0,15 \mathrm{a}$ & $0,19 \mathrm{a}$ & $0,18 \mathrm{a}$ & $0,24 \mathrm{a}$ & $0,17 \mathrm{a}$ \\
\hline CM - 2 & 0,15 a & $0,16 \mathrm{a}$ & $0,20 \mathrm{a}$ & $0,17 \mathrm{a}$ & $0,25 \mathrm{a}$ & $0,18 \mathrm{a}$ \\
\hline CM - 4 & $0,16 \mathrm{a}$ & $0,17 \mathrm{a}$ & $0,21 \mathrm{a}$ & $0,19 \mathrm{a}$ & $0,24 \mathrm{a}$ & $0,18 \mathrm{a}$ \\
\hline
\end{tabular}

ANP: antes do preparo do solo; APP: após o preparo do solo; SD: semeadura direta; PC: preparo convencional; CM: cultivo

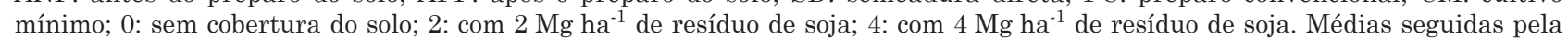
mesma letra minúscula, na mesma coluna, não diferem entre si pelo teste de Tukey a $5 \%$.

Quadro 3. Valores médios de cobertura do solo nos diferentes sistemas de preparo do solo, relativos aos níveis de resíduos vegetais

\begin{tabular}{|c|c|c|c|c|c|c|}
\hline \multicolumn{3}{|c|}{ SD } & $\mathrm{PC}$ & \multicolumn{3}{|c|}{$\mathbf{C M}$} \\
\hline \multicolumn{7}{|c|}{ Dose de resíduo vegetal $\left(\mathrm{Mg} \mathrm{ha}^{-1}\right)$} \\
\hline 0,0 & 2,0 & 4,0 & $0,0 \quad 2,0 \quad 4,0$ & 0,0 & 2,0 & 4,0 \\
\hline \multicolumn{7}{|c|}{ Cobertura do solo (\%) } \\
\hline 0,0 & 65,0 & 77,0 & $0,0 \quad 11,0 \quad 16,0$ & 0,0 & 14,0 & 23,0 \\
\hline
\end{tabular}

SD: semeadura direta; PC: preparo convencional; CM: cultivo mínimo. superiores aos do preparo convencional, devido, basicamente, ao efeito da incorporação parcial dos resíduos vegetais.

\section{Rugosidade da superfície do solo ao acaso}

Os valores do índice RR, determinados antes do preparo do solo, apresentaram variação de 3,31 a 5,41 mm (Quadro 4). Bertol et al. (2006) e Zoldan Junior et al. (2008), avaliando a rugosidade ao acaso sob diferentes condições de manejo do solo, obtiveram resultados semelhantes aos deste estudo.

As operações de preparo do solo aumentaram significativamente o valor da rugosidade ao acaso em

Quadro 4. Rugosidade da superfície do solo ao acaso nos diferentes momentos de avaliação dos tratamentos sob cultivo de soja

\begin{tabular}{|c|c|c|c|c|c|c|}
\hline Tratamento & ANP & APP & chuva 1 & chuva 3 & chuva 5 & chuva 7 \\
\hline \multicolumn{7}{|c|}{ Rugosidade ao acaso } \\
\hline $\mathrm{SD}-0$ & $4,10 \mathrm{Aa}$ & $4,10 \mathrm{Ad}$ & $4,01 \mathrm{~d}$ & $3,02 \mathrm{~d}$ & $2,48 \mathrm{~d}$ & $1,88 \mathrm{c}$ \\
\hline $\mathrm{SD}-2$ & $4,32 \mathrm{Aa}$ & $4,32 \mathrm{Ad}$ & $4,14 \mathrm{~d}$ & $3,82 \mathrm{~cd}$ & $3,69 \mathrm{~cd}$ & $3,44 \mathrm{bc}$ \\
\hline $\mathrm{SD}-4$ & $5,41 \mathrm{Aa}$ & 5,41 Acd & $4,57 \mathrm{~cd}$ & $4,69 \mathrm{~cd}$ & $4,52 \mathrm{bcd}$ & $4,49 \mathrm{~b}$ \\
\hline $\mathrm{PC}-0$ & $3,53 \mathrm{Ba}$ & $8,06 \mathrm{Ab}$ & $8,30 \mathrm{~b}$ & $7,18 \mathrm{~b}$ & $6,47 \mathrm{~b}$ & $5,74 \mathrm{~b}$ \\
\hline $\mathrm{PC}-2$ & $3,45 \mathrm{Ba}$ & 6,34 Acd & $6,99 \mathrm{~b}$ & $6,05 \mathrm{bc}$ & $5,22 \mathrm{bc}$ & $4,59 \mathrm{~b}$ \\
\hline $\mathrm{PC}-4$ & $3,31 \mathrm{Ba}$ & 7,27 Abc & $6,59 \mathrm{bc}$ & $5,65 \mathrm{bc}$ & $4,72 \mathrm{bcd}$ & $3,88 \mathrm{bc}$ \\
\hline CM - 0 & $4,29 \mathrm{Ba}$ & $15,41 \mathrm{Aa}$ & $13,61 \mathrm{a}$ & $11,59 \mathrm{a}$ & $10,05 \mathrm{a}$ & 8,99 a \\
\hline CM - 2 & $3,38 \mathrm{Ba}$ & $17,45 \mathrm{Aa}$ & $14,10 \mathrm{a}$ & $13,51 \mathrm{a}$ & $11,60 \mathrm{a}$ & $10,30 \mathrm{a}$ \\
\hline CM - 4 & $4,99 \mathrm{Ba}$ & $15,77 \mathrm{Aa}$ & $13,02 \mathrm{a}$ & $11,65 \mathrm{a}$ & $10,43 \mathrm{a}$ & $9,77 \mathrm{a}$ \\
\hline
\end{tabular}

ANP: antes do preparo do solo; APP: após o preparo do solo; SD: semeadura direta; PC: preparo convencional; CM: cultivo mínimo; 0: sem cobertura do solo; 2 : com $2 \mathrm{Mg} \mathrm{ha}^{-1}$ de resíduo de soja; 4: com $4 \mathrm{Mg}^{-1}$ de resíduo de soja. Médias seguidas pela mesma letra maiúscula, na linha, e minúscula, na coluna, não diferem entre si pelo teste de Tukey a $5 \%(C V=20,53 \%)$. 
comparação com o momento anterior ao preparo, como também constatado por Cogo et al. (1984), Eltz \& Norton (1997), Guzha et al. (2004), Castro et al. (2006) e Bertol et al. (2007, 2008).

O cultivo mínimo destacou-se dos demais sistemas de preparo estudados em relação ao aumento da rugosidade ao acaso, basicamente, por ter formado um grande número de torrões de dimensões maiores, por ação do escarificador.

Os valores do índice RR obtidos após o preparo do solo foram de 4,61, 7,22 e 16,21 mm para a semeadura direta, o preparo convencional e o cultivo mínimo, respectivamente, na média dos tratamentos de cada sistema de preparo. Esses valores são inferiores aos apresentados por Zobeck \& Onstad (1987), os quais obtiveram, em média, 6,80, 11,60 e 22,8 $\mathrm{mm}$ para semeadura direta, preparo convencional e cultivo mínimo, respectivamente, em diversos tipos de solos norte-americanos. Esses valores são também inferiores aos da escala de Renard et al. (1997), utilizados na RUSLE, considerando-se esses três sistemas de manejo do solo.

As operações de preparo do solo aumentaram a rugosidade ao acaso, na média dos tratamentos, em $110 \%$ no preparo convencional e em $297 \%$ no cultivo mínimo. Com relação ao efeito das operações de preparo na alteração da rugosidade da superfície do solo, justifica-se o menor efeito do preparo convencional em relação ao cultivo mínimo pela maior fragmentação dos agregados e torrões do solo; essa fragmentação é atribuída à ação repetida da grade niveladora, que promoveu maior destorroamento na superfície do solo sob preparo convencional.

Avaliando o efeito das chuvas sobre a redução da rugosidade ao acaso, verifica-se que, entre os tratamentos sob semeadura direta, o SD-0 foi o que apresentou a maior redução no valor do índice $R R$ com a aplicação das chuvas; no tratamento SD-4, por outro lado, a chuva não alterou de maneira significativa os valores de RR. No preparo convencional, no entanto, a aplicação das chuvas reduziu a rugosidade da superfície do solo até atingir uma condição semelhante à do microrrelevo existente no momento anterior ao preparo do solo. Isso deve-se, basicamente, ao efeito desagregador promovido pela ação do preparo secundário, promovendo, com isso, maior destruição dos agregados na superfície do solo neste sistema de preparo. Entretanto, em alguns casos, como nos tratamentos PC-0 e PC-2, pequena elevação no valor do índice de rugosidade ao acaso foi observada após a aplicação da primeira chuva simulada. Essa tendência também foi verificada por Eltz \& Norton (1997) e Govers et al. (2000), atribuindo-se esse fato à consolidação do solo pelo impacto das gotas de chuva e ao rearranjamento dos torrões e agregados do solo após terem sido umedecidos.

No cultivo mínimo, as maiores reduções do índice $\mathrm{RR}$ ocorreram com a incidência das primeiras chuvas sobre as parcelas, confirmando dados de Vidal Vázquez (2002), Castro et al. (2006), Bertol et al. (2007) e Zoldan Junior et al. (2008).

Com a aplicação das chuvas, totalizando $420 \mathrm{~mm}$ de lâmina de água, distribuídas em sete eventos de precipitação pluvial, a evolução dos valores do RR apresentou grande variabilidade de acordo com o tipo de preparo e nível de cobertura. Verificou-se que a relação entre os índices $\mathrm{RR}$ obtidos após a aplicação das sete chuvas e os obtidos após o preparo do solo foi igual a 0,46, 0,80 e 0,83 para os tratamentos SD-0, SD-2 e SD-4, respectivamente. Analisando esses valores, observa-se a importância da presença de cobertura vegetal na manutenção das propriedades físicas da superfície do solo em sistema de semeadura direta. No tratamento sob preparo convencional, o valor da referida relação foi, em média, de 0,65 e, no cultivo mínimo, de 0,60 , demonstrando que a semeadura direta foi o sistema mais estável quanto à variação do índice de rugosidade ao acaso, embora, na condição sem cobertura do solo, a variação de tal índice tenha sido mais expressiva. Essa melhor estabilidade estrutural do solo na semeadura direta está associada ao melhor condicionamento estrutural aos agregados do solo, em razão de ter sido mantido por longo período sem revolvimento. Em estudos dessa natureza, Cogo et al. (1984) observaram relação igual a 0,79 na semeadura direta, a 0,40 no preparo convencional e a 0,41 no cultivo mínimo. Também avaliando o mesmo tipo de relação, Guzha et al. (2004) encontraram valor de 0,78 para a semeadura direta, 0,34 para o preparo convencional e 0,57 para cultivo mínimo, após a aplicação de um volume acumulado de $408 \mathrm{~mm}$ de chuva artificial.

No preparo convencional, a aplicação das chuvas reduziu a rugosidade da superfície do solo até atingir uma condição semelhante à do microrrelevo existente no momento anterior ao preparo do solo. Isso deve-se, basicamente, ao efeito desagregador promovido pela ação do preparo secundário, mais intenso no preparo convencional do que no cultivo mínimo, promovendo, com isso, maior pulverização do solo. De maneira geral, verificou-se diminuição da rugosidade ao acaso, em todos os tratamentos, em função da aplicação das chuvas simuladas, corroborando estudos de Cogo et al. (1984), Eltz \& Norton (1997), Magunda et al. (1997), Castro et al. (2006), Bertol et al. (2007) e Zoldan Junior et al. (2008), realizados em diferentes tipos de solo.

Nos tratamentos submetidos às operações de preparo do solo, a aplicação das chuvas artificiais não proporcionou diferenças significativas entre os três níveis de cobertura do solo, considerando-se o mesmo sistema de preparo. Esse fato pode estar associado ao efeito da incorporação parcial dos resíduos vegetais, minimizando, dessa maneira, a capacidade deles de reduzir a ação das chuvas. No entanto, sob semeadura direta, observa-se que, na condição de máxima cobertura do solo (SD-4), a rugosidade ao acaso foi significativamente maior do que no tratamento sem 
cobertura do solo (SD-0), após a aplicação da sequência de chuvas.

\section{Relações entre a rugosidade ao acaso e o volume de chuvas}

Em todos os tratamentos, foram feitas avaliações do coeficiente de decaimento do índice RR, em decorrência da aplicação do volume de chuva acumulado. O modelo exponencial aplicado ajustouse bem aos valores de rugosidade da superfície do solo influenciados pelo volume de chuvas. Apresentou, em geral, coeficientes de determinação altos, assim como também ocorreu em estudos de Römkens \& Wang (1987), Eltz \& Norton (1997), Bertol et al. (2007) e Zoldan Junior et al. (2008).

Na semeadura direta (Figura 1), verificou-se que as chuvas simuladas não alteraram o valor do índice $R R$ na dose máxima de resíduo vegetal. Na dose de $2 \mathrm{Mg} \mathrm{ha}^{-1}$ de resíduo vegetal (SD-2), ocorreu diminuição da rugosidade ao acaso, ainda que pouco expressiva, ao passo que, na condição sem cobertura do solo (SD-0), as chuvas foram mais efetivas na redução do microrrelevo da superfície do solo. Esse comportamento é importante e serve para destacar a importância da presença do resíduo vegetal na proteção da superfície do solo contra o impacto das gotas de chuva e da força cisalhante do escoamento superficial.

Avaliando os valores dos coeficientes de decaimento da rugosidade, percebe-se que, para a semeadura direta sem cobertura do solo, o coeficiente de decaimento foi de 0,0018 , indicando redução de $0,00654 \mathrm{~mm}$ na rugosidade ao acaso para cada $\mathrm{mm}$ de chuva aplicada. No tratamento com valor médio de cobertura do solo, o coeficiente de decaimento foi de 0,0005 , representando redução na rugosidade ao acaso de 0,00204 $\mathrm{mm}$ para cada $\mathrm{mm}$ de chuva, enquanto no maior nível de cobertura o modelo de regressão exponencial utilizado indicou que as chuvas não influenciaram o valor do índice de rugosidade ao acaso.
Verifica-se, com isso, que na ausência de cobertura do solo a rugosidade ao acaso teve redução 3,2 vezes maior do que com 2,0 Mg ha-1 de resíduo vegetal de soja. Observa-se ainda que, embora as maiores doses de resíduo tenham favorecido a manutenção da rugosidade ao acaso, não foram eficientes em promover elevação na rugosidade inicial, possivelmente em razão da morfologia desse tipo de resíduo.

No preparo convencional (Figura 2), as linhas de tendência do modelo exponencial ajustado evidenciam diminuição da rugosidade ao acaso para os três níveis de cobertura do solo. Os valores do coeficiente de decaimento foram semelhantes entre os tratamentos sem cobertura do solo (PC-0) e com $2 \mathrm{Mg} \mathrm{ha}^{-1}$ de resíduo vegetal (PC-2), sendo estes ligeiramente inferiores aos do tratamento com $4 \mathrm{Mg} \mathrm{ha}^{-1}$ (PC-4). Isso não representou redução expressiva da rugosidade ao acaso, pois os valores caracterizaram uma diminuição de 0,00622, 0,00570 e 0,00942 $\mathrm{mm}$ da rugosidade por $\mathrm{mm}$ de chuva nas três doses de resíduo, respectivamente, em ordem crescente de cobertura do solo. Do ponto de vista prático, essas doses de resíduo são semelhantes quanto ao seu efeito na manutenção da rugosidade ao acaso. Dessa maneira, verifica-se que, neste tipo de preparo do solo, no qual a maior parte dos resíduos vegetais sofre incorporação, as diferentes doses de resíduo vegetal não influenciaram na manutenção da rugosidade da superfície do solo.

Ao comparar os tratamentos preparo convencional e semeadura direta, ambos com o mesmo percentual de cobertura por resíduo (Quadro 4), verificou-se que, na ausência de cobertura, o efeito da chuva na redução da rugosidade ao acaso foi semelhante nos dois sistemas de preparo. Isso demonstra que a semeadura direta, sem cobertura do solo, apresenta a mesma fragilidade que o preparo convencional em relação à manutenção do microrrelevo do solo.

No tratamento com valor intermediário de cobertura, a semeadura direta foi cerca de três vezes

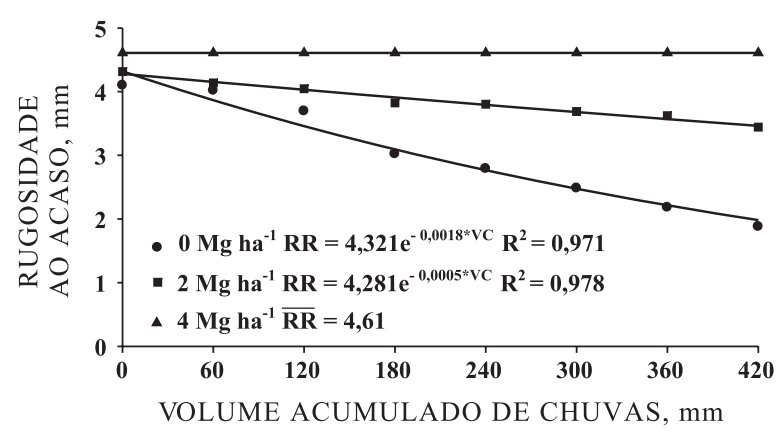

Figura 1. Estimativa da rugosidade ao acaso em função do volume acumulado de chuva sob semeadura direta, em diferentes níveis de cobertura do solo com resíduo vegetal de soja. *: significativo a $5 \%$ pelo teste $t$.

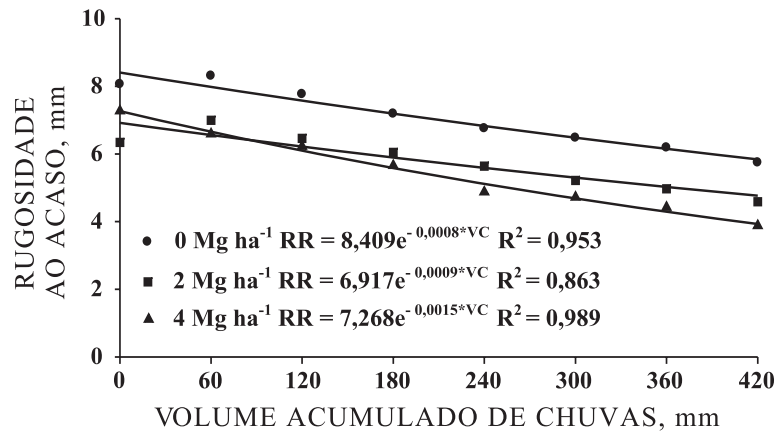

Figura 2. Estimativa da rugosidade ao acaso em função do volume acumulado de chuva sob preparo convencional em diferentes níveis de cobertura do solo com resíduo vegetal de soja. *: significativo a $5 \%$ pelo teste t. 
mais eficiente na manutenção da rugosidade ao acaso do que o preparo convencional, enquanto no tratamento com a maior percentagem de cobertura a referida eficiência chegou a ser de 6,5 vezes. Essa diferença da rugosidade em relação à energia das chuvas é importante, pois evidencia, ainda mais, os benefícios do sistema de semeadura direta, sob adequados níveis de cobertura do solo, no que se refere à manutenção da rugosidade do solo.

Sob cultivo mínimo (Figura 3), os valores dos coeficientes de decaimento, para os tratamentos CM0, CM-2 e CM-4, foram semelhantes, sendo, respectivamente, iguais a $0,0013,0,0012$ e 0,0010 , o que proporcionou as respectivas reduções de 0,01703 , 0,01732 e $0,01444 \mathrm{~mm}$ na rugosidade por $\mathrm{mm}$ de chuva aplicada, não havendo diferença significativa no decaimento da rugosidade superficial do solo entre os níveis de resíduo vegetal, neste sistema de preparo, com a aplicação das chuvas. Isso permite inferir que o efeito dos diferentes níveis de cobertura não influenciou na alteração da rugosidade ao acaso, devido, possivelmente, à incorporação parcial dos resíduos. Entretanto, deve-se ressaltar que, mesmo assim, no cultivo mínimo ocorreram os maiores valores de rugosidade após a aplicação das chuvas, em comparação com os outros sistemas, pois a escarificação promoveu a formação de torrões na superfície do solo, que não foram totalmente quebrados pela gradagem.

As reduções da rugosidade ao acaso, ocorridas no cultivo mínimo, foram mais acentuadas do que aquelas observadas no preparo convencional. Mesmo assim, verificou-se que a rugosidade era mais alta no cultivo mínimo do que no preparo convencional ao final dos testes de chuva, independentemente do nível de cobertura, por causa da escarificação praticada antes das chuvas, que elevou consideravelmente o microrrelevo do solo.

Os valores de coeficiente de decaimento da rugosidade da superfície do solo ao acaso, obtidos no

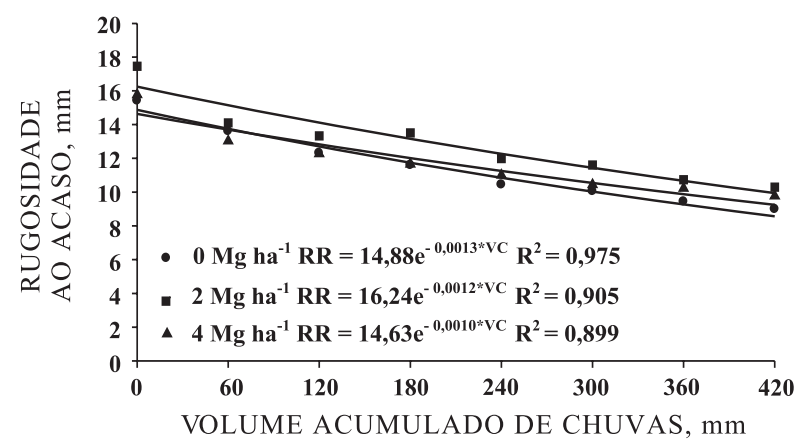

Figura 3. Estimativa da rugosidade ao acaso em função do volume acumulado de chuva sob cultivo mínimo, em diferentes níveis de cobertura do solo com resíduo vegetal de soja. *: significativo a $5 \%$ pelo teste $t$. cultivo mínimo, foram semelhantes aos obtidos por Bertol et al. (2007) e Zoldan Junior et al. (2008), em avaliação de diferentes sistemas de manejo de solo.

\section{CONCLUSÕES}

1. As operações de preparo do solo nos sistemas sob preparo convencional e cultivo mínimo aumentaram o valor do índice de rugosidade ao acaso em todas as doses de resíduo vegetal estudadas, em relação à condição anterior ao preparo do solo.

2. A operação de escarificação do solo, nos tratamentos submetidos ao cultivo mínimo, promoveu índices de rugosidade ao acaso superiores aos dos demais sistemas de preparo do solo.

3. A ação das chuvas na redução dos índices de rugosidade ao acaso foi mais expressiva nos tratamentos submetidos ao cultivo mínimo do que nos submetidos ao preparo convencional, em termos absolutos, embora, percentualmente, os valores tenham sido semelhantes nesses dois sistemas de preparo.

4. De maneira geral, a rugosidade da superfície do solo ao acaso relacionou-se inversamente com o volume de chuvas simuladas, pelo modelo de regressão exponencial.

\section{AGRADECIMENTO}

À Fundação de Desenvolvimento do Ensino, Ciência e Tecnologia do Estado do Mato Grosso do Sul (FUNDECT) pela concessão de bolsa e apoio financeiro ao desenvolvimento do projeto.

\section{LITERATURA CITADA}

ALLMARAS, R.R.; BURWELL, R.E. \& HOLT, R.F. Plow-layer porosity and surface roughness from tillage as affected by initial porosity and soil moisture at tillage time. Soil Sci. Soc. Am. J., 31:550-556, 1967.

ALVES SOBRINHO, T.; CARVALHO, D.F.; AQUINO, R.M. \& MONTEBELLER, C.A. Programa computacional para a definição de parâmetros hidráulicos utilizados na determinação da energia cinética da chuva simulada em infiltrômetro de aspersão. Eng. Rural, 12:28-35, 2001.

ALVES SOBRINHO, T.; FERREIRA, P.A \& PRUSKI, F.F. Desenvolvimento de um infiltrômetro de aspersão portátil. R. Bras. Eng. Agr. Amb.,.6:337-344, 2002.

ALVES SOBRINHO, T.; MACPHERSON, H.G. \& GÓMEZ, J.A. A portable integrated rainfall and overland flow simulator. Soil Use Manag., 24:163-170, 2008. 
BERTOL, I. Comprimento crítico de declive para preparos conservacionistas de solo. Porto Alegre, Universidade Federal do Rio Grande do Sul, 1995. 185p. (Tese de Doutorado)

BERTOL, I.; AMARAL, A.J.; VIDAL VÁZQUEZ, E.; PAZ GONZÁLEZ, A.; BARBOSA, F.T. \& BRIGNONI, L.F. Relações da rugosidade superficial do solo com o volume de chuva e com a estabilidade de agregados em água. $\mathrm{R}$. Bras. Ci. Solo, 30:543-553, 2006

BERTOL, I.; PAZ GONZÁLEZ, A. \& VIDAL VÁZQUEZ, E. Rugosidade superficial do solo sob diferentes doses de resíduo de milho submetido à chuva simulada. Pesq. Agropec. Bras., 42:103-110, 2007.

BERTOL, I.; ZOLDAN JUNIOR, W.A.; FABIAN, E.L.; ZAVASCHI, E.; PEGORARO, R. \& PAZ GONZÁLEZ, A. Efeito de escarificação e da erosividade de chuvas sobre algumas variáveis de valores de erosão hídrica em sistemas de manejo de um Nitossolo Háplico. R. Bras. Ci. Solo, 32:747-757, 2008.

BURWELL, R.E.; ALLMARAS, R.R. \& AMEMIYA, M.A. Field measurement of total porosity and surface microrelief of soils. Soil Sci. Soc. Proc., 27:697-700, 1963.

CASTRO, L.G.; COGO, N.P. \& VOLK, L.B.S. Alterações na rugosidade superficial do solo pelo preparo e pela chuva e sua relação com a erosão hídrica. R. Bras. Ci. Solo, 30:339$352,2006$.

COGO, N.P. Effect of residue cover, tillage induced roughness, and slope length on erosion and related parameters. West Lafayette, Purdue University, 1981. 346p. (Tese de Doutorado)

COGO, N.P.; MOLDENHAUER, W.C. \& FOSTER, G.R. Soil loss reductions from conservation tillage practices. Soil Sci. Soc. Am. J., 48:368-373, 1984.

ELTZ, F.L.F. \& NORTON, L.D. Surface roughness changes as affected by rainfall erosivity, tillage, and canopy cover. Soil Sci. Soc. Am. J., 61:1746-1755, 1997.

EMPRESA BRASILEIRA DE PESQUISA AGROPECUÁRIA EMBRAPA. Serviço Nacional de Levantamento e Conservação de solo. Manual de métodos de análise de solo. Rio de Janeiro, Ministério da Agricultura, 1997. 212p.

GOMES, A.C.S. Efeito de diferentes estratégias de irrigação sob a cultura da soja (Glycine $\max (\mathrm{L}$.) Merril) na região de Santiago, RS. Universidade Federal de Santa Maria. Santa Maria, RS. 2007. 132p. (Dissertação de Mestrado)

GOVERS, G.; TAKKEN, I. \& HELMING, K. Soil roughness and overland flow. Agronomie, 20:131-146, 2000.

GUZHA, A.C. Effects of tillage on soil microrelief, surface depression storage and soil water storage. Soil Till. Res., 76:105-114, 2004.
KAMPHORST, E.C.; JETTEN, V.; GUÉRIF, J.; PITKANEN, J.; IVERSEN, N.V.; DOUGLAS, J.T. \& GONZÁLES, A.P Predicting depressional storage from soil surface roughness. Soil Sc. Soc. Am. J., 64:1749-1758, 2000.

KEMPER, W.D. \& ROSENAU, R.C. Aggregate stability and size distribution. In: KLUTE, A. Methods of soil analysis, Part 1. Phisical and mineralogical methods. 2ed. 1986. p.425-441. (Agronomy Monograph, 9)

MAGUNDA, M.K.; LARSON, W.E.; LINDEN D.R. \& NATER, E.A. Changes in microrelief and their effects on infiltration and erosion during simulated rainfall. Soil Technol., 10:57-67, 1997.

ONSTAD, C. A. Depressional storage on tilled soil surfaces. Trans. Am. Soc. Agric. Eng., 27:729-732, 1984.

RENARD, K.G.; FOSTER, G.R. \& WEESIES, G.A. Predicting soil erosion by water: A guide to conservation planning with the revised universal soil loss equation (RUSLE). Washington, USDA, 1997. 384p. (Agricultural Handbook, 703).

RÖMKENS, M.J.M. \& WANG, J.Y. Effect of tillage on soil roughness. Trans. Am. Soc. Agric. Eng., 29:429-433, 1986.

RÖMKENS, M.J.M. \& WANG, J.Y. Soil roughness changes from rainfall. Trans. Am. Soc. Agric. Eng., 30:101-107, 1987.

TACONET, O. \& CIARLETTI, V. Estimating soil roughness indices on a ridge-and-furrow surface using stereo photogrammetry. Soil Till. Res., 93:64-76, 2007.

VIDAL VÁZQUEZ, E. Influencia de la precipitación y el laboreo en la rugosidad del suelo y la retención de agua en microdepresiones. La Coruña, Universidade de Coruña, 2002. 430p. (Tese de Doutorado)

ZOBECK, T.M. \& POPHAM, T.W. Modification of the wind erosion roughness index by rainfall. Soil Till. Res., 42:4761, 1997.

ZOBECK, T.M. \& ONSTAD, C.A. Tillage and rainfall effects on random roughness: A review. Soil Till. Res., 9:1-20, 1987.

ZOLDAN JUNIOR, W.A. Rugosidade superficial do solo ocasionada por uma operação de escarificação aplicada após cinco anos e meio de uso de sistemas de manejo do solo, e suas relações com a erosividade da chuva e erosão hídrica em um Nitossolo Háplico. Lages, Universidade do Estado de Santa Catarina, 2006. 100p. (Tese de Mestrado)

ZOLDAN JUNIOR, W.A.; BERTOL, I.; PEGORARO, R.; FABIAN, E.L.; ZAVASCHI, E. \& VIDAL VÁZQUEZ. E. Rugosidade superficial do solo formada por escarificação e influenciada pela erosividade da chuva. R. Bras. Ci. Solo, 32:353-362, 2008. 
